\title{
Redescripción de una especie y descripción de dos especies nuevas de Acanthococcus Signoret (Hemiptera, Eriococcidae) de la región Neotropical
}

\author{
Patricia González ${ }^{1}$ \& Lucía E. Claps ${ }^{1,2}$
}

\begin{abstract}
'Instituto Superior de Entomología "Dr. Abraham Willink” (INSUE), Facultad de Ciencias Naturales e Instituto Miguel Lillo, Universidad Nacional de Tucumán y CIUNT. Miguel Lillo 205. 4000, Tucumán, Argentina. mopagon2004@yahoo.com.ar

${ }^{2}$ Facultad de Ciencias y Tecnología, sede Diamante, Universidad Autónoma de Entre Ríos. Urquiza y Tratado del Pilar. 3105, Diamante, Entre Ríos, Argentina.luciaclaps@arnet.com.ar
\end{abstract}

\begin{abstract}
Redescription of one species and description of two new species of Acanthococcus Signoret (Hemiptera, Eriococcidae) from the Neotropical Region. The adult female and the first-instar nymph of Acanthococcus piptadeniae (Hempel), from Brazil, are redescribed and illustrated, and two new species from Argentina are described and illustrated based on the adult female. A key to Acanthococcus species with six setae on the protibiae and five on meso- and metatibiae, including Acanthococcus dubius (Cockerell), cited in the Neotropical Region, is presented.
\end{abstract}

KEYWORDS. Argentina; Brazil; Eriococcus; taxonomic key.

RESUMEN. Redescripción de una especie y descripción de dos especies nuevas de Acanthococcus Signoret (Hemiptera: Eriococcidae) de la región Neotropical. Se redescriben e ilustran la hembra adulta y la ninfa del primer estadio de Acanthococcus piptadeniae (Hempel), de Brasil y se describen e ilustran dos especies nuevas de la Argentina basado en la hembra adulta. Se presenta una clave para las especies de Acanthococcus con seis setas en las protibias y cinco en meso y metatibias, incluyendo a Acanthococcus dubius (Cockerell), citada para la región Neotropical.

PALABRAS CLAVE. Argentina; Brasil; clave; Eriococcus

En la región Neotropical se registran 24 géneros y 72 especies de Eriococcidae (Foldi \& Kozár 2007; Kozár \& Konczné Benedicty 2008; BenDov et al. 2010; Hodgson \& Miller 2010). Cook \& Gullan (2004), en su análisis molecular y Hodgson \& Miller (2010), en su revisión de los géneros sudamericanos de la familia, consideran que las especies neotropicales previamente ubicadas en Eriococcus Targioni Tozzetti, 1868, están más apropiadamente ubicadas en Acanthococcus Signoret, 1875, género representado por 29 especies en esta región (Hodgson \& Miller 2010). Entre esas especies, A. dubius (Cockerell, 1896) está citada para Venezuela y Brasil (Foldi \& Kozar 2007) y A. piptadeniae (Hempel, 1937) para Brasil.

Un carácter importante a considerar en la taxonomía de este género es el número de setas presentes en las tibias, el cual varía de cuatro a seis. Este estudio incluye cuatro especies de Acanthococcus que presentan seis setas en las tibias protorácicas y cinco setas en las tibias meso y metatorácicas. El objetivo de este trabajo es aportar al conocimiento de la diversidad de eriocóccidos de la región Neotropical.

\section{MATERIAL Y MÉTODOS}

El material empleado proviene de las colecciones del Instituto Biológico de São Paulo, Brasil (IBSP) y de la
Fundación-Instituto Miguel Lillo, Tucumán, Argentina (IMLA). Las especies $A$. dubius y $A$. arctostaphyli no fueron observadas, las características de ambas fueron tomadas de Miller \& Miller (1992).

Las técnicas empleadas para efectuar los preparados permanentes son las usadas comúnmente para "cochinillas" (Granara de Willink 1990). Los dibujos representan en la mitad derecha la superficie ventral y en la mitad izquierda la superficie dorsal; los detalles de algunas estructuras alrededor del dibujo central no guardan proporción entre sí. Se empleó un tubo de cámara clara de microscopio óptico, de distintos aumentos.

Las medidas se expresan en milímetros ( $\mathrm{mm})$ y en micrómetros $(\mu \mathrm{m})$. En la especie redescripta, el rango corresponde a todos los ejemplares estudiados, en las especies nuevas las medidas son la del holotipo y entre paréntesis el rango de los valores de los paratipos, aún en los casos en que las medidas de holo y paratipo coincidan. Los microconductos se designan siguiendo a González (2008), el "tipo A" (Fig. 2) con el vestíbulo ancho, dividido longitudinalmente por un tabique, el extremo interno en forma de dos protuberancias y el orificio dermal simple. El microconducto "tipo B" (Fig. 1) presenta el vestíbulo angosto, no dividido, el extremo interno redondeado y el orificio dermal simple. 


\section{RESULTADOS}

Acanthococcus gracielae González \& Claps sp. nov.

(Fig. 1)

Material tipo. Holotipo: hembra adulta ubicada a la derecha en el preparado, marcado con un círculo rojo, con las siguientes medidas: $1,7 \mathrm{~mm}$ de largo y $1,0 \mathrm{~mm}$ de ancho. Argentina, Salta, Río Colorado $\left(24^{\circ} 48^{\prime} \mathrm{S} ; 62^{\circ} 27^{\prime} \mathrm{O}\right)$, hospedero desconocido, 06.xi.1993, M.C.G. Willink col. (IMLA). Paratipos: 3 hembras adultas con los mismos datos que el holotipo; a la hembra ubicada a la izquierda en el mismo preparado del holotipo se suma un preparado con 2 hembras adultas (IMLA).

Diagnosis. Abundantes setas cónicas que forman líneas transversas en los segmentos abdominales y torácicos; macroconductos simétricos y microconductos "tipo B" numerosos en ambas superficies; setas marginales no diferenciadas de las dorsales; metacoxas con aproximadamente 50 poros, 30 dorsales y 20 ventrales; lóbulos anales con dos setas ventrales.

Descripción. Hembra adulta: contorno del cuerpo ovalado; longitud $1,7(1,8-2,0) \mathrm{mm}$ y ancho $1,0(0,8-1,3) \mathrm{mm}$. Lóbulos anales alargados, esclerosados; longitud lóbulos 78 (65-72) $\mu \mathrm{m}$ y ancho 54 (36-55) $\mu \mathrm{m}$; con cuatro microconductos y tres setas cónicas en la superficie dorsal, la interna superior más pequeña, de 31 (33-38) $\mu \mathrm{m}$, la interna inferior de $51(36-55) \mu \mathrm{m}$ y la externa de $58(50-53) \mu \mathrm{m}$; dos setas flageladas en la superficie ventral, la inferior de 84 (96-100) $\mu \mathrm{m}$ y la superior de $48(57-70) \mu \mathrm{m}$ de longitud; seta apical de 240 (234-240) $\mu \mathrm{m}$. Placa media ausente.

Superficie dorsal: con abundantes setas cónicas, derechas, con extremo apenas redondeado, de distinto tamaño, entre 21-60 (21-60) $\mu \mathrm{m}$, forman líneas paralelas a la segmentación en todos los segmentos, las de mayor tamaño se ubican hacia el margen del cuerpo y en la región cefálica. Numerosos macroconductos simétricos en toda la superficie, de 19-29 (19-29) $\mu \mathrm{m}$ de longitud y 4-5 (4-5) $\mu \mathrm{m}$ de ancho, con conducto interno con glándula terminal. Microconductos "tipo B", de 5-7 (5-7) $\mu \mathrm{m}$ de longitud y 2 (2) $\mu \mathrm{m}$ de ancho, distribuidos en la superficie y en el margen; microconductos "tipo A" muy escasos en la superficie. Anillo anal con ocho setas de $108(108-120) \mu \mathrm{m}$ de longitud y una vuelta de poros.

Margen: setas marginales no diferenciadas de las dorsales.

Superficie ventral: con setas flageladas, de distinta longitud, entre 24-72 (24-72) $\mu \mathrm{m}$, en toda la superficie. Setas agrandadas, de menor tamaño que las dorsales, submarginales, una por segmento abdominal, más numerosas en tórax y región cefálica. Seta suranal flagelada, de 60 (60-72) $\mu \mathrm{m}$ de longitud. Microespinas numerosas en zona media y mediolateral de todos los segmentos abdominales. Poros quinqueloculares de 5 (5) $\mu \mathrm{m}$ de diámetro, escasos a lo ancho de todos los segmentos abdominales y cercanos a los espiráculos; poros triloculares escasos dispersos en región cefálica, zona media del tórax, cerca de espiráculos y en los

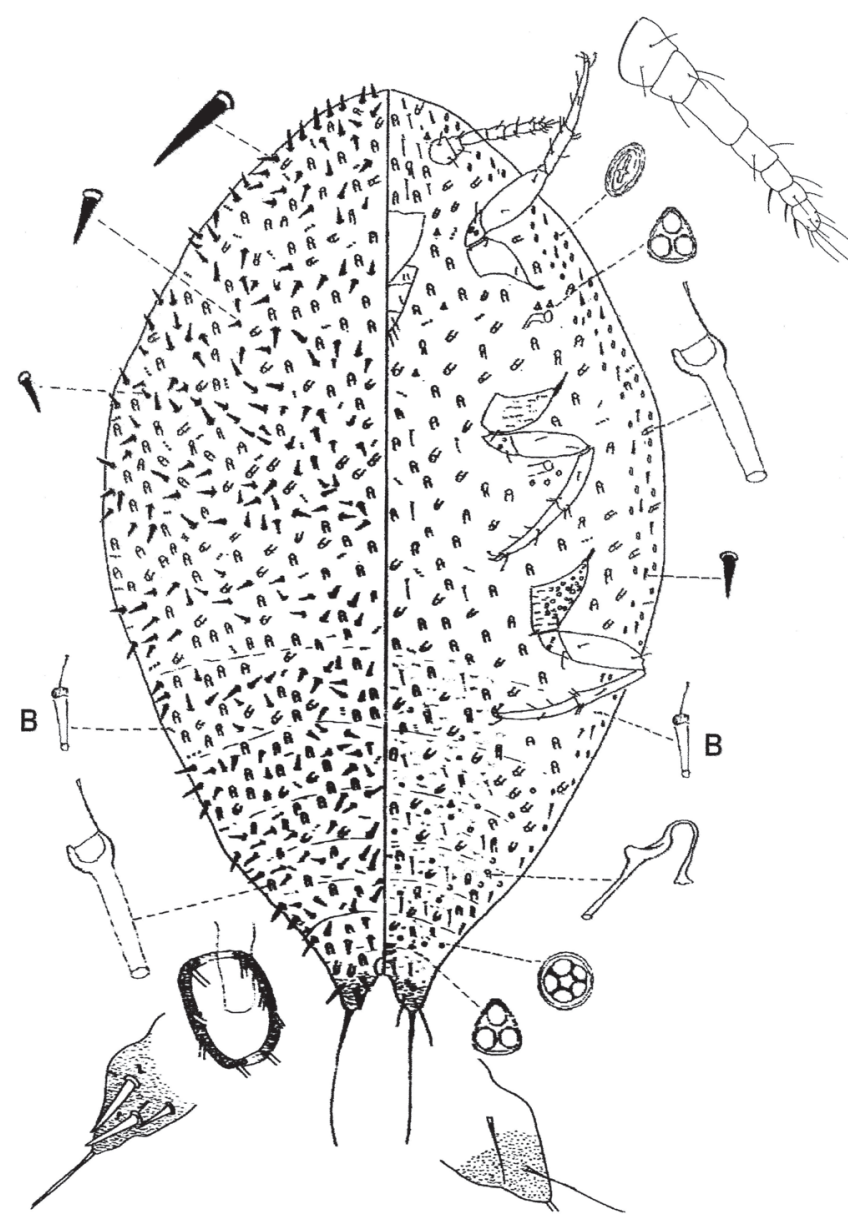

Fig. 1. Acanthococcus gracielae sp. nov. Hembra adulta. (B: microconducto "tipo B").

segmentos abdominales. Poros cruciformes submarginales en las regiones cefálica y torácica, llegan aproximadamente hasta el segmento abdominal V. Macroconductos simétricos, similares en tamaño a los dorsales, numerosos en toda la superficie; algunos conductos más angostos, de 2 (2) $\mu \mathrm{m}$ de ancho y 17-19 (17-19) $\mu \mathrm{m}$ de longitud y de taza ancha, en el margen y zona media en todos los segmentos abdominales y centrales en el metatórax. Microconductos "tipo B", similares a los dorsales, dispersos en la superficie. Patas bien desarrolladas; coxas protorácicas de 125 (132-150) $\mu \mathrm{m}$ de longitud; fémur más trocánter de 177 (180-199) $\mu \mathrm{m}$; tibia de 96 (96-103) $\mu \mathrm{m}$, con seis setas; tarso de $110(110-115) \mu \mathrm{m}$, uña de 24 (24-36) $\mu \mathrm{m}$ de longitud. Patas mesotorácicas con coxas de 125 (137-151) $\mu \mathrm{m}$ de longitud, con microespinas ventrales en parte proximal y media; fémur más trocánter de 180 (197-211) $\mu \mathrm{m}$; tibia de 91 (120-125) $\mu \mathrm{m}$, con cinco setas; tarso de 112 (112-125) $\mu \mathrm{m}$; uña de 29 (29-36) $\mu \mathrm{m}$ de longitud. Patas metatorácicas con coxas de 167 (156-175) $\mu \mathrm{m}$ de longitud, con microespinas y aproximadamente 50 poros, 30 dorsales y 20 ventrales, cribados e irregulares; trocánter más fémur 214 (202-226) $\mu \mathrm{m}$ de longitud; fémur con cinco setas, sin poros; tibia de $136(136-148) \mu \mathrm{m}$, con cinco setas; tarso de 144 (144-156) $\mu \mathrm{m}$; uña con dentículo; 
digitígulas tarsales y digitígulas ungueales iguales entre sí, con extremos apenas ensanchados. Antenas de 199 (201-213) $\mu \mathrm{m}$ de largo, de siete segmentos, el tercero sin setas y de mayor longitud, el resto de los segmentos con setas flageladas; con setas carnosas en los últimos tres segmentos. Lóbulos frontales ausentes. Labium trisegmentado, primer segmento con dos pares de setas, segundo segmento con un par y tercer segmento con cinco pares de setas. Tubo anal esclerosado.

Distribución. Argentina: Salta. Eco-región Chaco seco.

Comentarios. Acanthococcus gracielae sp. nov. se asemeja a Acanthococcus granarae sp. nov., A. piptadeniae, A. dubius y A. arctostaphyli Ferris, 1955, esta última de la región Neártica, por presentar seis setas en las tibias protorácicas y cinco en las tibias meso y metatorácicas.

Acanthococcus granarae sp. nov. se diferencia de $A$. gracielae sp. nov. en los siguientes puntos (caracteres de $A$. gracielae entre paréntesis): (i) setas dorsales con extremo agudo (con extremo apenas redondeado), (ii) poros quinqueloculares y triloculares abundantes en abdomen (escasos),(iii) lóbulos anales con cuatro setas ventrales (dos) y lóbulos frontales presentes (ausentes). De acuerdo con Miller \& Miller (1992), A. dubius se diferencia de A. gracielae (caracteres de $A$. gracielae entre paréntesis) por: (i) setas con extremo agudo, ordenadas en bandas longitudinales (con extremo apenas redondeado, ordenadas en líneas paralelas a la segmentación), (ii) tres setas ventrales en los lóbulos anales (dos) y (iii) abundantes poros quinqueloculares en el abdomen (escasos). Acanthococcus arctostaphyli se diferencia de $A$. gracielae (caracteres de $A$. gracielae entre paréntesis) por: (i) setas marginales diferenciadas de las dorsales (no diferenciadas), (ii) setas grandes dorsales dispuestas en tres líneas longitudinales (setas grandes hacia el margen del cuerpo y en región cefálica), (iii) cuatro setas ventrales en los lóbulos anales (dos) y (iv) poros quinqueloculares numerosos en segmentos abdominales (escasos). Acanthococcus piptadeniae se diferencia de $A$. gracielae en los siguientes puntos (caracteres de $A$. gracielae entre paréntesis): (i) tres setas ventrales en los lóbulos anales (dos), (ii) metacoxas con 610 poros (aproximadamente 50 poros) y (iii) placa media presente (ausente).

Etimología. El epíteto específico de la especie, gracielae, está dedicado a la Dra. Graciela Esteban, por brindarle permanente apoyo y ayuda a la primera autora.

\section{Acanthococcus granarae González \& Claps sp. nov.} (Fig. 2)

Material tipo. Holotipo: hembra adulta ubicada a la izquierda del preparado, marcado con un círculo rojo, con las siguientes medidas: 2,9 $\mathrm{mm}$ de largo y 1,9 $\mathrm{mm}$ de ancho.

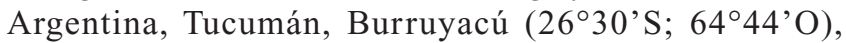
hospedero desconocido, 6.xi.1995, M.C.G. Willink col. Paratipo: una hembra adulta con los mismos datos de recolección, en la misma lámina (IMLA).

Diagnosis. Setas dorsales con extremo agudo, de mayor tamaño en una banda submarginal a lo largo del cuerpo;

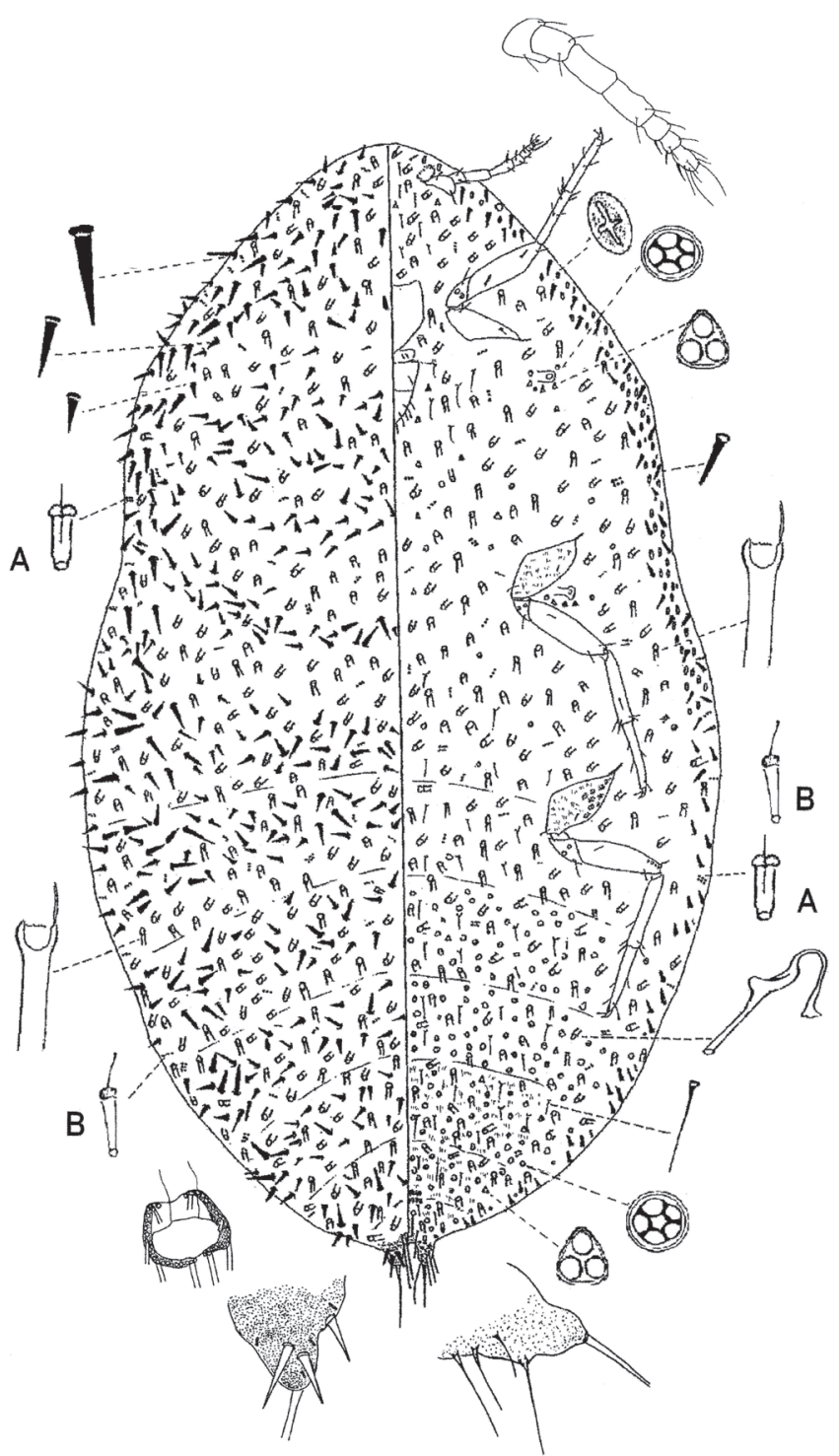

Fig. 2. Acanthococcus granarae sp. nov. Hembra adulta (A: microconducto "tipo A"; B: microconducto "tipo B").

microconductos "tipos A y B" en ambas superficies; poros quinqueloculares abundantes en abdomen, poros triloculares numerosos en tórax, región cefálica y cerca de espiráculos; poros cruciformes abundantes en margen y submargen de regiones cefálica y torácica; metacoxas con aproximadamente 40 poros, 20-25 dorsales y 10-15 ventrales; lóbulos frontales presentes y lóbulos anales con cuatro setas ventrales.

Descripción. Hembra adulta: contorno del cuerpo ovalado; longitud 2,9 (2,7) mm y ancho 1,9 (1,8) mm. Lóbulos anales esclerosados; longitud lóbulos 107 (84) $\mu \mathrm{m}$ y ancho 78 (40) $\mu \mathrm{m}$; superficie dorsal con cuatro microconductos y tres setas cónicas, las internas superior e inferior de 50 (48) $\mu \mathrm{m}$, la externa de $70(70) \mu \mathrm{m}$; superficie ventral con cuatro setas flageladas, entre 38-112 (38-112) $\mu \mathrm{m}$ de longitud; seta apical cortada en el holotipo (240) $\mu \mathrm{m}$ de longitud. Placa media ausente.

Superficie dorsal: con abundantes setas cónicas, angostas, con extremo agudo, de distinto tamaño, entre 29-68 (29-68) 
$\mu \mathrm{m}$, forman tres o cuatro líneas de setas paralelas a la segmentación en cada segmento; las setas de mayor tamaño forman una banda submarginal (dos a cinco setas por segmento) a lo largo del cuerpo. Macroconductos simétricos, de 7(7) $\mu \mathrm{m}$ de ancho y 15-24 (15-24) $\mu \mathrm{m}$ de longitud, abundantes en toda la superficie. Microconductos "tipo A", de 3 (3) $\mu \mathrm{m}$ de ancho y 7 (7) $\mu \mathrm{m}$ de longitud y "tipo B", de 2 (2) $\mu \mathrm{m}$ de ancho y 7-9 (7-9) $\mu \mathrm{m}$ de longitud, abundantes en toda la superficie. Anillo anal con ocho setas de $103 \mu \mathrm{m}$ de longitud (cortadas en el paratipo) y una vuelta de poros.

Margen: setas marginales no diferenciadas de las dorsales.

Superficie ventral: con setas flageladas, numerosas, de distinta longitud, de 30-132 (30-132) $\mu \mathrm{m}$, en la línea media de toda la superficie, en el abdomen forman líneas paralelas a la segmentación; las setas más largas en la zona media del abdomen y entre las antenas. Setas agrandadas del mismo tipo que las dorsales a lo largo del margen pero ausentes en la zona cefálica, entre las antenas. Seta suranal flagelada, de 72 (76) $\mu \mathrm{m}$ de longitud. Microespinas en segmentos abdominales VI-VIII, en área media y mediolateral. Poros quinqueloculares de 5-6 (5-6) $\mu \mathrm{m}$ de diámetro, abundantes en segmentos abdominales IV-VIII y escasos cerca de los espiráculos; poros triloculares numerosos en zona media y mediolateral del tórax, cercanos a los espiráculos y en región cefálica, escasos en el abdomen. Poros cruciformes abundantes en margen y submargen de las regiones cefálica y torácica, llegan pocos hasta el segmento abdominal V. Macroconductos similares en tamaño a los dorsales, abundantes y dispersos en toda la superficie; macroconductos angostos de 2 (2) $\mu \mathrm{m}$ de ancho y 12 (12) $\mu \mathrm{m}$ de longitud y taza ancha, numerosos en la zona submedia del tórax y abdomen. Microconductos "tipos A y B", abundantes en toda la superficie. Patas bien desarrolladas, largas y delgadas. Patas protorácicas con coxas de $180(211) \mu \mathrm{m}$ de longitud; fémur más trocánter de 264 (259) $\mu \mathrm{m}$; tibia de 151(161) $\mu \mathrm{m}$, con seis setas; tarso de 144 (144) $\mu$ m; uña de 33 (29) $\mu \mathrm{m}$ de longitud. Patas mesotorácicas con coxas de $192(177) \mu \mathrm{m}$ de longitud, con microespinas; fémur más trocánter 259 (276) $\mu \mathrm{m}$; tibia de 180 (180) $\mu \mathrm{m}$, con cinco setas; tarso de 156 (153) $\mu \mathrm{m}$; uña de 36 (39) $\mu \mathrm{m}$ de longitud. Patas metatorácicas con coxas de mayor tamaño que los otros pares, de 225 (215) $\mu \mathrm{m}$ de longitud, con microespinas y poros translúcidos grandes y cribados, aproximadamente 40 (40), 20-25 dorsales y 10-15 ventrales; trocánter más fémur de 304 (303) $\mu \mathrm{m}$ de longitud; fémur con pocos poros translúcidos y cinco setas; tibia de 196 (196) $\mu \mathrm{m}$, con cinco setas; tarso de 196 (186) $\mu \mathrm{m}$; uña con dentículo; digitígulas tarsales y digitígulas ungueales iguales entre sí, delgadas y con extremos apenas expandidos. Antenas de 313 (274) $\mu \mathrm{m}$ de longitud, de siete segmentos, tercero y cuarto de igual longitud, el tercero sin setas, el resto de los segmentos con setas flageladas; con setas carnosas en los últimos tres segmentos. Lóbulos frontales presentes, de tamaño semejante al primer segmento antenal. Labium trisegmentado, el primer segmento con dos pares de setas, segundo con un par y tercer segmento con cinco pares de setas. Tubo anal esclerosado.
Distribución. Argentina: Tucumán. Eco-región Chaco seco.

Comentarios. Acanthococcus granarae sp. nov. es semejante a $A$. dubius, $A$. piptadeniae, A. gracielae sp. nov. y A. arctostaphyli.

Acanthococcus dubius se diferencia de A. granarae (caracteres de $A$. granarae entre paréntesis) en los siguientes puntos: (i) setas dorsales forman bandas longitudinales (forman líneas paralelas a la segmentación del cuerpo), (ii) un tipo de microconducto (dos tipos) y (iii) lóbulos frontales ausentes (presentes). Acanthococcus piptadeniae se diferencia de $A$. granarae (caracteres de $A$. granarae entre paréntesis) por: (i) tres setas ventrales en los lóbulos anales (cuatro), (ii) placa media presente (ausente) y (iii) lóbulos frontales ausentes (presentes). Acanthococcus arctostaphyli se diferencia de $A$. granarae (caracteres de $A$. granarae entre paréntesis) por: (i) setas con ápice redondeado o truncado (con ápice agudo) y las setas dorsales de los lóbulos anales de aproximadamente igual tamaño (seta externa de mayor longitud). Las diferencias de $A$. granarae con $A$. gracielae ya fueron tratadas en los comentarios de esta última.

Etimología. El epíteto específico, granarae, está dedicado a la Dra. María Cristina Granara de Willink, reconocida coccidóloga de la región Neotropical.

\section{Acanthococcus piptadeniae (Hempel)}

\section{(Fig. 3)}

Eriococcus piptadeniae Hempel, 1937: 6-8; Lepage 1938: 380; Silva et al. 1968: 160.

Eriococcus piptandeniae; Hoy 1963: 109 (enmienda injustificada); Miller \& Gimpel 2000: 301; Kozár 2009: 100; Ben-Dov et al. 2010 (catálogo electrónico) (Nombre erróneo de especie).

Eriococcus peptadeniae; Claps 1993: 7 (Nombre erróneo de especie). Acanthococcus piptandeniae; Miller \& Gimpel 1996: 603; Hodgson \& Miller 2010: 99 (Nombre erróneo de especie y cambio de combinación).

Material tipo. Brasil, São Paulo, Itatinga; 17.i.1935; sobre Piptadenia falcata (Fabaceae); A. Hempel col. Holotipo hembra depositado en Instituto Biológico de São Paulo, Brasil, No 710 (Hempel 1937).

Material examinado. En la etiqueta izquierda se lee Eriococcus peptadeniae Hempel sp. nov. Coll. A. Hempel. R710. En la etiqueta derecha: Gav. 19. Div. 39. (6 hembras adultas + una ninfa del primer estadio). IBSP.

Diagnosis. Setas dorsales cónicas con extremo agudo; microconductos "tipos A y B" en ambas superficies; poros triloculares numerosos en regiones cefálica, torácica y abdominal; poros cruciformes numerosos en los márgenes de la región cefálica y en tórax; metacoxas con aproximadamente 10 poros, 6-10 dorsales y $0-3$ ventrales; lóbulos anales con tres setas ventrales.

Redescripción. Hembra adulta: contorno del cuerpo ovalado; longitud 1,8-2,4 mm y ancho 0,9-1,4 mm. Lóbulos anales esclerosados, longitud 49-62 $\mu \mathrm{m}$ y ancho $39-49 \mu \mathrm{m}$; con tres setas cónicas en la superficie dorsal, seta interna inferior de 48-50 $\mu \mathrm{m}$, la interna superior de 28-30 $\mu \mathrm{m}$ y seta externa de 41-43 $\mu \mathrm{m}$. Superficie ventral con tres setas 
flageladas, de 83-140 $\mu \mathrm{m}$ de longitud; seta apical de 228$253 \mu \mathrm{m}$. Placa media observada en dos ejemplares.

Superficie dorsal: con numerosas setas cónicas, con extremo agudo, de distinto tamaño, 15-64 $\mu \mathrm{m}$; forman dos o tres líneas de setas paralelas a la segmentación en cada segmento abdominal y torácico, más numerosas en región cefálica. Macroconductos simétricos, de 5-7 $\mathrm{mm}$ de ancho y aproximadamente $25 \mathrm{~mm}$ de longitud, abundantes en toda la superficie. Microconductos de dos tipos, numerosos en toda la superficie; el "tipo A" de 2,5 $\mu \mathrm{m}$ de ancho y 5,0 $\mu \mathrm{m}$ de longitud; el "tipo B" de 1,5 $\mu \mathrm{m}$ de ancho y 5-7 $\mu \mathrm{m}$ de longitud. Anillo anal con ocho setas de 72-84 $\mu \mathrm{m}$ de longitud y una vuelta de poros.

Margen: setas marginales no diferenciadas de las dorsales.

Superficie ventral: con setas flageladas, numerosas, de distinta longitud $28-72 \mu \mathrm{m}$, en segmentos abdominales y entre antenas. Setas agrandadas ausentes. Seta suranal flagelada, de 65-66 $\mu \mathrm{m}$ de longitud. Microespinas escasas en segmentos abdominales posteriores. Poros quinqueloculares de $5 \mu \mathrm{m}$ de diámetro, numerosos en los segmentos abdominales posteriores, dispersos en los anteriores, cercanos a los espiráculos y en región frontal; poros triloculares numerosos en región cefálica, parte media del tórax y cerca de los espiráculos, dispersos en abdomen. Poros cruciformes numerosos en el margen y submargen de las regiones cefálica y torácica. Macroconductos de tamaño semejante a los del dorso, numerosos y dispersos en toda la superficie; conductos de menor tamaño, de $2 \mu \mathrm{m}$ de ancho y $12 \mu \mathrm{m}$ de longitud, con taza ancha, numerosos en la zona submedia del tórax y en abdomen. Microconductos "tipos A y B", de tamaño semejante a los del dorso, numerosos en la superficie. Patas bien desarrolladas. Patas protorácicas con coxas de 132-134 $\mu \mathrm{m}$; trocánter más fémur de 180-211 $\mu \mathrm{m}$ de longitud; tibia de 90-110 $\mu \mathrm{m}$, con seis setas; tarso de 105-134 $\mu \mathrm{m}$; uña de 26-33 $\mu \mathrm{m}$ de longitud. Patas mesotorácicas con coxas de 136$163 \mu \mathrm{m}$ de longitud, con microespinas; trocánter más fémur 207-230 $\mu \mathrm{m}$; tibia de 108-142 $\mu \mathrm{m}$, con cinco setas; tarso de 119-149 $\mu \mathrm{m}$; uña de 31-36 $\mu \mathrm{m}$ de longitud. Patas metatorácicas con coxas de 139-168 $\mu \mathrm{m}$ de longitud, con microespinas y 10 poros, 6-10 dorsales y $0-3$ ventrales; trocánter más fémur de 211-259 $\mu \mathrm{m}$; tibia de 123-156 $\mu \mathrm{m}$, con cinco setas, tarso de 132-156 $\mu \mathrm{m}$, uña de 33-37 $\mu \mathrm{m}$ de longitud, con dentículo; digitígulas tarsales y digitígulas ungueales iguales entre sí, delgadas y con extremos apenas expandidos. Antenas de 209-237 $\mu \mathrm{m}$ de longitud, con siete segmentos, el tercero sin setas, el resto de los segmentos con setas flageladas; con setas carnosas en los últimos tres segmentos. Lóbulos frontales ausentes. Labium trisegmentado, primer segmento con dos pares de setas, segundo con un par y tercer segmento con seis pares de setas. Tubo anal esclerosado.

Distribución: Brasil: São Paulo.

Comentarios. Acanthococcus piptadeniae es semejante a A. dubius, A. arctostaphyli, A. gracielae sp. nov. y A. granarae sp. nov.

Acanthococcus dubius se diferencia de A. piptadeniae (caracteres de $A$. piptadeniae entre paréntesis) en los siguientes puntos: (i) un solo tipo de microconducto (dos tipos), (ii) an-

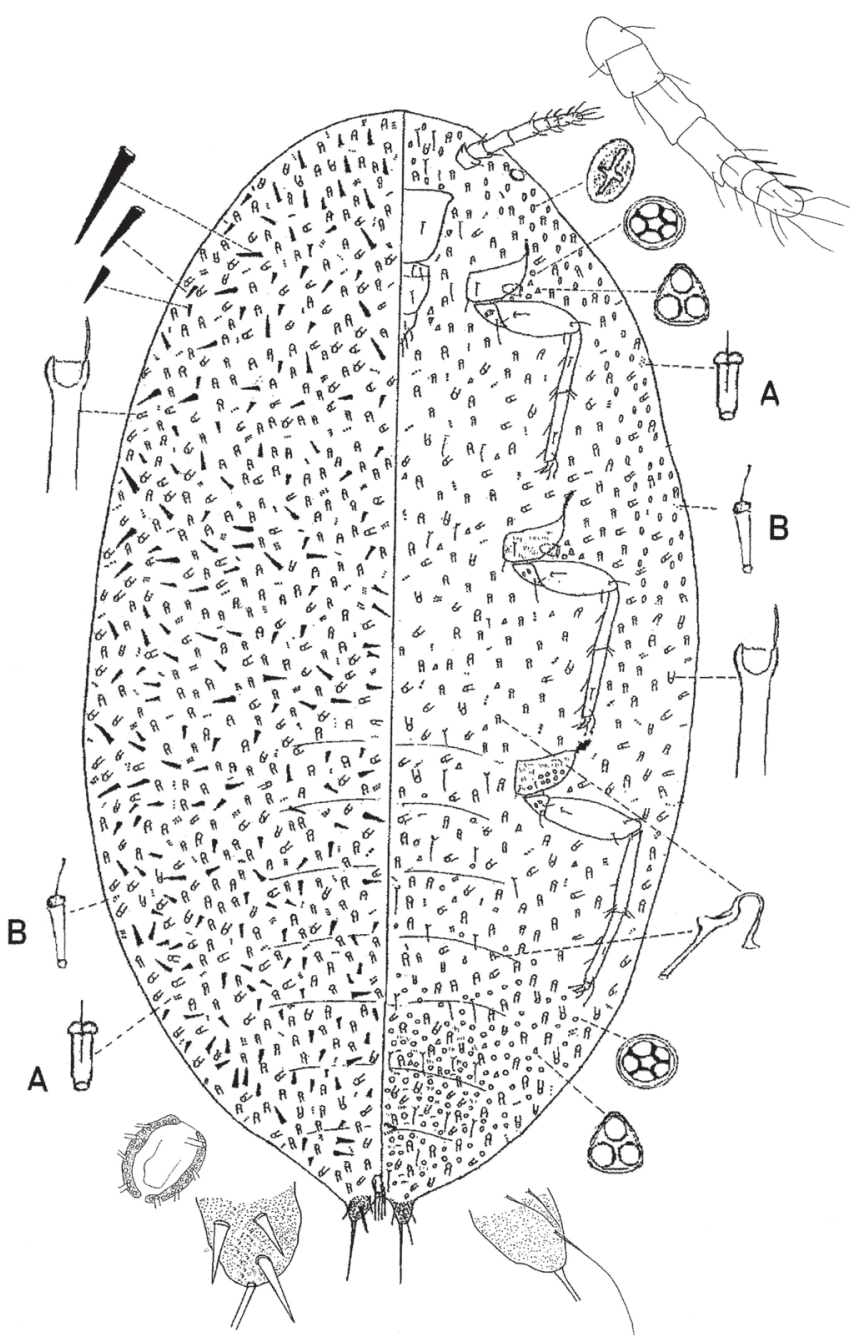

Fig. 3. Acanthococcus piptadeniae (Hempel). Hembra adulta. (A: microconducto" tipo A"; B: microconducto "tipo B").

tenas con seis segmentos (siete) y (iii) metacoxas con 13-38 poros (6-10 poros). Acanthococcus arctostaphyli se diferencia de $A$. piptadeniae (caracteres de $A$. piptadeniae entre paréntesis) por: (i) setas cónicas con extremo redondeado (con extremo agudo), (ii) metacoxas con 16-96 poros dorsales y 024 ventrales (6-10 dorsales y $0-3$ ventrales) y (iii) lóbulos anales con cuatro setas ventrales (tres). Las diferencias de $A$. piptadeniae con $A$. gracielae y $A$. granarae ya fueron tratadas en los comentarios de estas dos últimas especies.

\section{Ninfa del primer estadio (Fig. 4)}

Material examinado. En la etiqueta de la izquierda se lee Eriococcus peptadeniae Hempel sp. nov. Typo. Coll. A. Hempel. R710. En la etiqueta derecha: Gav. 19. Div. 38. (aproximadamente 90 ninfas del primer estadio). IBSP.

Redescripción: contorno del cuerpo ovalado; longitud 346-455 $\mu \mathrm{m}$ y ancho 188-217 $\mu \mathrm{m}$. Lóbulos anales de 24$29 \mu \mathrm{m}$ de longitud y 14-21 $\mu \mathrm{m}$ de ancho, con tres setas cónicas en la superficie dorsal, seta interna superior de 27-43 $\mu \mathrm{m}$; interna inferior de $24-36 \mu \mathrm{m}$ y la externa de $17-24 \mu \mathrm{m}$ de 


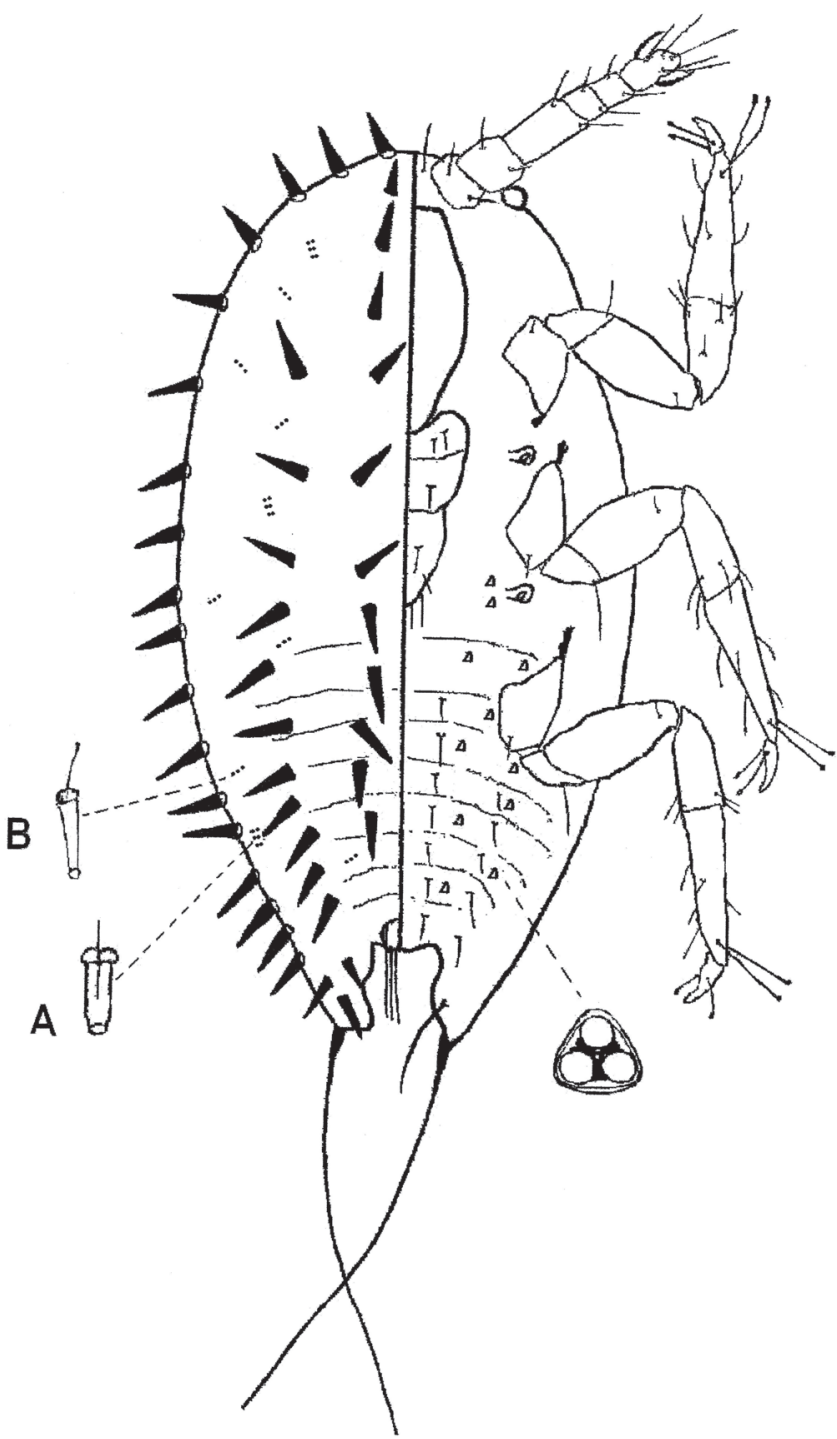

Fig. 4. Acanthococcus piptadeniae (Hempel). Ninfa del primer estadio. (B: microconducto “tipo B”).

longitud; una seta flagelada en la superficie ventral de $52-$ $63 \mu \mathrm{m}$ y seta apical de 120-216 $\mu \mathrm{m}$ de longitud.

Superficie dorsal: con setas cónicas, con extremo agudo, entre 14-29 $\mu \mathrm{m}$ de longitud, forman líneas longitudinales de 11 setas cada una, un par medial y un par lateral; las últimas ausentes en las regiones cefálica y protorácica; las mediales ausentes en los tres últimos segmentos abdominales. Microconductos "tipos A y B", escasos en la superficie. Anillo anal con ocho setas de 60-69 $\mu \mathrm{m}$ de longitud y una vuelta de poros.

Margen: 36 setas en total, a lo largo del margen del cuerpo, una seta en cada segmento abdominal, cada una de 19-24 $\mu \mathrm{m}$ de longitud.

Superficie ventral: con setas flageladas, de distinta longitud 12-29 $\mu \mathrm{m}$, en zona media de los segmentos abdominales y un par entre las antenas. Seta suranal flagelada, de 36-45 $\mu \mathrm{m}$ de longitud. Poros quinqueloculares ausentes; poros triloculares, uno o dos pares por segmento abdominal, dos poros cercanos a cada coxa metatorácica y a los espiráculos posteriores. Microconductos ausentes. Patas bien desarrolladas, tibia de menor longitud que el tarso, uña con dentículo; digitígulas tarsales y digitígulas ungueales iguales entre sí, delgadas y con extremos apenas expandidos. Tibia protorácica con seis setas. Antenas de 96-120 $\mu \mathrm{m}$ de longitud, con seis segmentos, el tercero más largo, todos con setas, el último segmento con setas carnosas. Lóbulos frontales ausentes. Labium trisegmentado, el primer segmento con dos pares de setas, segundo con un par y tercer segmento con dos pares de setas.

Observaciones. En aproximadamente 90 ejemplares analizados no se pudo observar claramente el anillo anal por lo cual el mismo no fue ilustrado

Nota: posteriormente a la descripción original de $A$. piptadeniae, varios autores, incluídos en la sinonimia del presente trabajo, citan incorrectamente el epíteto específico de esta especie y el nombre genérico del hospedero. Esto se debe a la enmienda injustificada del epíteto específico realizada por Hoy (1963), quien al parecer, pensaba que el nombre correcto del hospedero era Piptandenia falcata. De hecho, el nombre correcto del hospedero es Piptadenia falcata. Asimismo cabe aclarar que en los rótulos de las preparaciones permanentes tanto de hembra adulta y de ninfas del primer estadio se lee "Eriococcus peptadeniae", siendo el nombre válido dado en la descripción original de Hempel "Eriococcus piptadeniae".

Clave para las especies de Acanthococcus con seis setas en las tibias protorácicas, presentes en la región Neotropical

1. Setas dorsales forman líneas longitudinales submarginales, mediolaterales y mediales A. dubius (Cockerell)

1 '. Setas dorsales forman líneas paralelas a la segmentación del cuerpo 2

2. Lóbulos anales con dos setas ventrales, poros quinqueloculares escasos, microconductos "tipo B" predominantes, “tipo A” escasos ....A. .gracielae González \& Claps sp. nov.

2 '. Lóbulos anales con mayor número de setas ventrales, poros quinqueloculares numerosos, microconductos "tipos A y B" numerosos 3

3. Lóbulos frontales ausentes, lóbulos anales con tres setas ventrales, metacoxas con 6-10 poros, placa media presente A. piptadeniae (Hempel)

3'. Lóbulos frontales presentes, lóbulos anales con cuatro setas ventrales, metacoxas con aproximadamente 40 poros, placa media ausente

A. granarae González \& Claps sp.nov.

\section{CONCLUSIONES}

Siguiendo a Cook \& Gullan (2004) y Hodgson \& Miller (2010), consideramos las cuatro especies aquí presentadas pertenecientes al género Acanthococcus. Las mismas comparten una serie de caracteres: igual número de setas en las tibias, setas dorsales cónicas, macroconductos simétricos, 
conductos angostos y de taza ancha en la superficie ventral y lóbulos anales con tres setas dorsales. Difieren entre sí por el número de setas ventrales en los lóbulos anales, el tipo de microconductos, la presencia o ausencia de lóbulos frontales y de placa media.

En la especie de Hempel 1937, Acanthococcus piptadeniae, se revalida el epíteto específico correcto, piptadeniae, en reemplazo de piptandeniae, empleado por varios autores.

\section{AGRADECIMIENTOS}

Al Dr. Sergio Ide del Instituto Biológico de São Paulo, Brasil por el préstamo del material y al Instituto Superior de Entomología “Dr. Abraham Willink", Tucumán, Argentina por solventar los gastos de campaña. A dos árbitros anónimos por sus oportunos comentarios.

\section{REFERENCIAS}

Ben-Dov, Y.; D. R. Miller \& G. A. P. Gibson. 2010. Scale Net: a Database of the Scale insects of the world. Disponible en: http://www.sel.barc. usda.gov/scalenet/scalenet.htm (acessado 5 junio 2010).

Claps, L. E. 1993. Lista de tipos y cotipos de Coccoidea depositados en colecciones entomológicas de instituciones de Brasil. The Scale 18: $2-12$.

Cockerell, T. D. 1896. Preliminary diagnoses of new Coccidae. Psyche, Supplement 7: 18-21.

Cook, L. G. \& P. J. Gullan. 2004. The gall-inducing habit has evolved multiple times among the eriococcid scale insects (Sternorrhyncha: Coccoidea: Eriococcidae). Biological Journal of the Linnean Society 83: 441-452.

Foldi, I. \& F. Kozár. 2007. New species and new records of Eriococcus (Hemiptera: Coccoidea, Eriococcidae) from South America. Zootaxa 1573: 51-64.
González, P. 2008. Two new species of Eriococcus (Hemiptera: Coccoidea: Eriococcidae from the Andino Patagonica region of Argentina, with a key to the Eriococcus species of that region. Zootaxa 1899: 50-56.

Granara de Willink, M. C. 1990. Conociendo nuestra fauna 1. Superfamilia Coccoidea (Homoptera: Sternorryncha.). Serie Monográfica y Didáctica 6. Facultad de Ciencias Naturales e Instituto Miguel Lillo. UNT: 1-43.

Hempel, A. 1937. Novas especies de Coccideos (Homoptera) do Brasil. Archivos do Instituto Biologico 8: 5-10.

Hodgson, C. \& D. R. Miller. 2010. A review of the Eriococcid genera (Hemiptera: Sternorrhyncha: Coccoidea) of South America. Zootaxa 2459: 1-101.

Hoy, J. M. 1963. A Catalogue of the Eriococcidae of the World. New Zealand Department of Scientific and Industrial Research Bulletin 150, 260 p.

Kozár, F. 2009. Zoogeographical analysis and status of knowledge of the Eriococcidae (Hemiptera), with a world list of species. Bolletino di Zoologia agraria e di Bachicoltura, Ser. II, 41: 87-121.

Kozár, F \& Z. Konczné Benedicty. 2008. Description of three new genera, five new species and some additional data on the taxonomy and distribution of Neotropic Eriococcidae (Homoptera: Coccoidea: Eriococcidae). Bolletino di Zoologia agraria e di Bachicoltura, Ser. II, 40: 117-144.

Lepage, H. S. 1938. Catalogo dos coccideos do Brasil. Revista do Museu Paulista, São Paulo, 23: 327-491.

Miller, D. R. \& M. E. Gimpel. 1996. Nomenclatural changes in the Eriococcidae (Homoptera: Coccoidea). Proceedings of the Entomological Society of Washington 98: 597-606.

Miller, D. R. \& M. E. Gimpel. 2000. A Systematic Catalogue of the Eriococcidae (felt scales) (Hemiptera: Coccoidea) of the world. Andover, Intercept Limited, $589 \mathrm{p}$.

Miller, D. R. \& G. L. Miller. 1992. Systematic Analysis of Acanthococcus (Homoptera: Cocoidea: Eriococcidae) in the Western United States. Transactions of the American Entomological Society 118: 1-106.

Silva, A.; G. A. d'Araujo; C. R. Gonçalves; D. M. Galvão; A. J. L.Gonçalves; J. Gomes; M. N. Silva \& L. Simoni. 1968. Quarto catalogo dos insetos que vivem nas plantas do Brasil. Insetos, hospedeiros e inimigos naturais. Rio de Janeiro, Ministério da Cultura, vol. 1, 622 p.

Signoret, V. 1875. Essai sur les cochenílles ou gallinsectes, $14^{\circ}$ partie (1). Annales de la Societe Entomologique de France 5: 15-40.

Recibido 10/6/2010; aceptado 15/4/2011

Editor: Daniela Maeda Takiya 